1

2

\title{
Towards the Mechanical Characterization of Abdominal Wall by Inverse Analysis
}

\author{
R. Simón-Allué ${ }^{a, *}$, B. Calvo ${ }^{\mathrm{a}, \mathrm{b}}$, A. A. Oberai ${ }^{\mathrm{c}}$, P. E. Barbone ${ }^{\mathrm{d}}$ \\ a Aragón Institute of Engineering Research (I3A), Universidad de Zaragoza, Spain. \\ ${ }^{\mathrm{b}}$ Centro de Investigación Biomédica en Red en Bioingeniería, Biomateriales y \\ Nanomedicina (CIBER-BBN), Spain. \\ ${ }^{\mathrm{c}}$ Mechanical, Aerospace and Nuclear Engineering, Rensselaer Polytechnic \\ Institute, Troy NY, USA \\ ${ }^{\mathrm{d}}$ Mechanical Department, Boston University, Boston MA, USA
}

\begin{abstract}
The aim of this study is to characterize the passive mechanical behaviour of abdominal wall in vivo in an animal model using only external cameras and numerical analysis. The main objective lies in defining a methodology that provides in vivo information of a specific patient without altering mechanical properties. It is demonstrated in the mechanical study of abdomen for hernia purposes. Mechanical tests consisted on pneumoperitoneum tests performed on New Zealand rabbits, where inner pressure was varied from $0 \mathrm{mmHg}$ to $12 \mathrm{mmHg}$. Changes in the external abdominal surface were recorded and several points were tracked. Based on their coordinates we reconstructed a 3D finite element model of the abdominal wall, considering an incompressible hyperelastic material model defined by two parameters. The spatial distributions of these parameters (shear modulus and non linear parameter) were calculated by inverse analysis, using two different types of regularization: Total Variation Diminishing (TVD) and Tikhonov $\left(H^{1}\right)$. After solving the inverse problem, the distribution of the material parameters were obtained along the abdominal surface. Accuracy of the results was evaluated for the last level of pressure.

Results revealed a higher value of the shear modulus in a wide stripe along the craneo-caudal direction, associated with the presence of linea alba in conjunction with fascias and rectus abdominis. Non linear parameter distribution was smoother and the location of higher values varied with the regularization type. Both regularizations proved to yield in an accurate predicted displacement field, but $H^{1}$ obtained a smoother material parameter distribution while TVD included some discontinuities. The methodology here presented was able to characterize in vivo the passive non linear mechanical response of the abdominal wall.
\end{abstract}

Key words: Abdominal wall; Mechanical properties; In-vivo tests; Patient specific behavior; Inverse analysis; Nonlinear elasticity imaging. 


\section{Introduction}

The most common solution for severe hernia cases is implanting a surgical mesh to cover the zone where the hernia takes place. Since an abdominal hernia consists of an opening in the abdominal wall, this mesh has to replace the function of the muscle/fascia while it is healing, absorbing strain and stress in lieu of the tissue. This technique, known as the Lichtenstein's tension-free mesh procedure, has been widely applied since its introduction (Lichtenstein et al., 1989). However, some common complications are still frequently associated with this surgery, such as pain (Paajanen \& Hemunen, 2004), inflammation (Klinge et al., 2002), hernia relapse or even mesh breakage (Cobb et al., 2005).

Previous studies have been focused on the mechanical behavior of the abdominal wall. Most of those authors have worked with in vitro tests, mainly uniaxial or biaxial experiments, either on animal models (Hernández et al., 2011; Lyons et al., 2014; Cooney et al., 2015) or using human samples (Kirilova et al., 2011; Martins et al., 2012; Ben Abdelounis et al., 2013). Other authors performed ex vivo tests on human tissues (Tran et al., 2014; Podwojewski et al., 2014), analysing the response of human abdomen when pressure is applied to the whole wall and to isolated layers. Few of the studies dealt with human abdomen in in vivo conditions. Song et al. (2006) and Szymczak et al. (2012) both estimated strain values in specific directions when the patient was subjected to usual activities: expiration, bending and torsion of the abdomen, (Szymczak et al., 2012) and during a surgery when a pneumoperitoneum was induced (Song et al., 2006). More recently, Tran et al. (2016) also performed measurements of the elasticity and local stiffness of abdominal wall by shear wave elastography, determining the active and passive linear elastic mechanical response of the wall under physiological activities.

The main benefit of an in vivo test lies in the possibility of measuring a living tissue without altering its natural response, in a non-invasive way. Moreover, this type of test provides information about a specific specimen, which can be very useful for the patient-specific treatment. Over the last two decades, great strides have been made to enable the in vivo measurement of tissue deformation, via techniques referred to as "elastography" (Ophir et al., 1991; Rivaz et al., 2013; Chauvet et al., 2015; Tran et al., 2016). This fact, combined with the parallel advance in efficient algorithms, has made possible the in vivo mechanical characterization of several soft tissues by inverse analysis: human liver (Nava et al., 2008), breast tissue (Goenezen et al., 2012) and thyroid gland (Kybic \& Smutek, 2005). With few exceptions (e.g. (Goenezen et al., 2012)) most work in this area has focused on linear elastic, small strain tissue characterization.

\footnotetext{
* Corresponding author. Mechanical Department. c/ María de Luna s/n 50018. Zaragoza. Spain.
} Email address: rsimon@unizar.es, Tel.: +34 876555187 
In this work we present an estimation of both linear and nonlinear elastic properties of the abdominal wall measured under in vivo conditions. Taking inflation tests performed on an animal model as a starting point, displacement of different points of the wall were measured by photogrammetry and the whole abdominal cavity was reconstructed. This methodology was previously introduced in Simón-Allué et al. (2015). The identification of the mechanical properties from the displacement field together with the inner pressure constitutes a nonlinear inverse problem. To solve it, we have employed an efficient algorithm that uses a gradient based quasi-Newton minimization strategy to seek those material parameter distribution whose numerical displacement field better match the measured displacement field (Gokhale et al., 2008; Goenezen et al., 2011).

The objective of this study is to establish a methodology able to characterize in vivo abdominal tissue only by the use of cameras and FE simulation of the mechanical response through a 3D model of the wall. A long term goal of this work is to enable patient specific mechanical property characterization to improve surgical treatment planning.

\section{Material and Methods}

\subsection{Experimental tests}

Experimental tests were conducted on an animal model, specifically New Zealand White rabbits, frequently used as a model in hernia studies (Nilsson, 1982; Bellón, 2007; Hernández et al., 2011). This animal model presents the benefits of being easy to handle and biologically very sensitive to non-biocompatible materials. For this study 2 rabbits were subjected to several pneumoperitoneum tests, following the protocol described in (Simón-Allué et al., 2015).

Animals were obtained from the Animal Experimentation Service of the Research Support Services of the University of Zaragoza, with an average weight of $2.20 \mathrm{~kg}$. Animals were healthy and free of clinically observable diseases. Prior to the procedure, they were kept under stable conditions of light and temperature following the recommendations given by the "Guide for the Care and Use of Laboratory Animals" (GuideCare, 2011). All procedures were carried out under Project Licence 01/11 approved by the in-house Ethics Committee for Animal Experiments of the University of Zaragoza.

Before each experiment, animals were shaved from front to rear legs and placed face up on a surgical table, so that the abdominal surface remained well exposed. Thereafter, the skin was spotted with black dots situated in a grid pattern. These points were used as fiducial points in the post-processing. Concurrently, a synchronized stereo rig composed of two Prosilica GT1290 cameras was situated above the animal to record the deformation of the external abdomen during the pneumoperitoneum (see Fig. 1 (a)).

Subsequently, a Verres needle was inserted in the lower abdomen and connected to a Standard 
Karl Storz endoscope at the other end. A schematic diagram of the whole setup is shown in Fig. 1 (b). Gas (carbon dioxide) was introduced through this needle increasing the intra abdominal pressure (IPA) from $0 \mathrm{mmHg}$ to $12 \mathrm{mmHg}$, in steps of $1 \mathrm{mmHg}$. Following each test, gas pressure was reduced back to zero. This procedure was repeated 4 times per specimen. The first test was excluded from the analysis to account for preconditioning. For the numerical postprocessing, 2 valid cycles were analyzed per animal. Images of the abdominal wall at the initial and final moment of the experiment can be seen in Fig. 2.

From the video recorded by the cameras, a pair of frames was extracted corresponding to each level of pressure. Frames were analyzed using the digital PhotoModeler (2013) Software, which allows us to determine the three dimensional coordinates of points shown in both images using photogrammetry. In this way the three dimensional coordinates of the black dots painted on the surface of the abdomen were determined as a function of the abdominal pressure. For this geometry and camera resolution an accuracy better than 1mm can be assured (Simón-Allué et al., 2015).

\subsection{Numerical analysis}

The measured displacement of the black dots was used to determine the spatial distribution of the material properties of the abdomen. This inverse problem was solved by minimizing the difference between the measured and a predicted displacement field, where the latter was required to satisfy the equations of equilibrium for a nonlinear hyperelastic material. The spatial distribution of the material properties in this model was varied so as to yield a predicted displacement field that was optimally close to the measured displacement. A gradient-based optimization algorithm (LBFGS) (Zhu et al., 1997) was used to solve this problem, and the gradients were determined efficiently through the use of adjoint equations. At every step of the optimization algorithm one forward non linear elastic problem, and one adjoint elastic problem was solved.

In the following sections, we first describe the forward problem and then describe the inverse problem.

\subsubsection{Nonlinear forward problem}

Abdominal wall muscles are composed of individual components known as muscle fibres surrounded by connective tissue (ground substance, collagen and elastin fibres in different proportions) capable of absorbing the muscle lengthening. The connective tissue is largely responsible for transmitting forces. In this paper we consider only the passive properties of muscle tissue since it determines the response registered during the pneumoperitoneum with the rabbit anesthetized. The abdominal wall is subjected to large deformations with negligible volume changes, that is, only isochoric $(J \approx 1)$ motions are possible. This response is modelled with an incompressible hyperelastic model. Due to the preferred directions of orientation of collagen and muscular fibers, the abdominal wall may display an anisotropic stress response when each 
muscle layer is modelled (Hernández et al., 2011). However, when the response of several layers working together is analysed, the level of anisotropy decreases significantly (Hernández et al., 2011). Therefore, we model the tissue using an incompressible, isotropic, and nonlinear elastic model depending on two parameters, whose spatial variation may provide different mechanical response of the tissue zones.

We characterize the hyperelastic response through the second Piola-Kirchhoff stress tensor given by

$$
\mathbf{S}=J p \mathbf{C}^{-1}+2 \frac{\partial \Psi(\overline{\mathbf{C}})}{\partial \mathbf{C}}
$$

where $J$ is the Jacobian of the deformation gradient $\left(\mathbf{F}=\frac{\partial \mathbf{x}}{\partial \mathbf{X}}\right), p$ the hydrostatic pressure associated with the additional volumetric constraint $J-1=0, \mathbf{C}$ the right Cauchy-Green strain tensor $\left(\mathbf{C}=\mathbf{F}^{T} \mathbf{F}\right)$ and $\overline{\mathbf{C}}$ the modified Cauchy-Green strain tensor defined as $\overline{\mathbf{C}}=J^{-\frac{2}{3}}$ C. $\Psi$ is a convex strain energy density function (SEF) dependent on $\bar{I}_{1}$, the first invariant of $\overline{\mathrm{C}}$.

We use the following SEF

$$
\Psi(\overline{\mathbf{C}})=\frac{\mu}{2 \gamma}\left(e^{\gamma\left(\bar{I}_{1}-3\right)}-1\right)
$$

with an exponential stress-strain response the based on the Blatz model (Blatz et al., 1969), able to reproduce the hyperelastic response found in Simón-Allué et al. (2015).

This SEF contains two independent parameters: $\mu$ is the shear modulus at zero strain and $\gamma$ is a non linear parameter which determines the nonlinearity of the material response. These two material parameters have clear, distinct, physical interpretations: $\mu$ governs the small strain behavior independent of the value of $\gamma$, while $\gamma$ controls the exponential stiffening, independent of $\mu$. To observe the respective roles of these two parameters a sensitivity analysis with a computational simulation of biaxial tests was performed. We plot in Fig. 3 the Cauchy stressstretch curves varying one parameter while the other one remains fixed: variation of $\mu$ (Fig. 3 (a)) and variation of $\gamma$ (Fig. $3(\mathrm{~b})$ ). The response becomes more rigid with the increasing values of $\gamma$ and $\mu$.

For this specific material model defined by Eq. 2, the second Piola-Kirchhoff stress is given by

$$
\mathbf{S}=p J \mathbf{C}^{-1}+\mu\left(\mathbf{I}-\frac{1}{3} \bar{I}_{1} \mathbf{C}^{-1}\right) e^{\gamma\left(\bar{I}_{1}-3\right)}
$$

The Cauchy stress tensor $\boldsymbol{\sigma}$ is $1 / J$ times the push-forward of $\mathbf{S}$, and for this SEF is given by 


$$
\sigma=p \mathbf{I}+\mu J^{-1}\left(\overline{\mathbf{b}}-\frac{1}{3} \bar{I}_{1} \mathbf{I}\right) e^{\gamma\left(\bar{I}_{1}-3\right)}
$$

where $\overline{\mathbf{b}}=J^{-\frac{2}{3}} \mathbf{b}$, and $\mathbf{b}=\mathbf{F F}^{T}$ the left Cauchy-Green tensor.

\subsubsection{Nonlinear inverse problem}

The solution of the finite element inverse problem for incompressible materials was fully discussed in detail in Goenezen et al. (2011). In this section we provide a brief review of basic concepts, necessary for understanding the results obtained. First, a finite element model to simulate the pneumoperitoneum tests is created, assuming nominal values for the material properties of the abdominal wall. Assuming the same values along the surface entails an homogeneous response of the wall. Then, an inverse problem is solved interactively by minimizing an objective function under the constraint that the predicted displacement fields satisfy the forward problem. This objective function measures the difference between the predicted and the measured displacement fields. This difference is driven to a minimum by iteratively updating the spatial distribution of the material properties through a quasi-Newton algorithm. The gradient is efficiently computed through the use of an adjoint equation (Gokhale et al., 2008; Goenezen et al., 2011).

The objective function is defined as follows

$$
\Pi=\frac{1}{2} \sum_{i=1}^{n_{\text {meas }}} w_{i}\left\|\mathbf{u}_{i}-\mathbf{u}_{\text {meas }}^{i}\right\|_{0}^{2}+\frac{1}{2} \sum_{j=1}^{N_{\beta}} \alpha_{j} R\left(\beta_{j}\right)
$$

where $\mathbf{u}_{\text {meas }}^{i}$ are the measured displacements fields obtained by photogrammetry, $\mathbf{u}^{i}$ are the predicted displacements fields and $n_{\text {meas }}$ is the number of independent measured displacement fields. The vector $\beta_{j}=\left[\beta_{1}, \beta_{2}\right]=[\mu, \gamma]$ is the vector of material coefficients required to describe the abdominal muscle material. The spatial distribution of these parameters is determined by solving the optimization problem, where the optimization variables are the values of these material parameters at the integration points. Further, $\|\cdot\|_{0}$ denotes the $L_{2}$ norm and $w_{i}$ are weighting factors selected to ensure that the contributions to the objective function from all measurements are of the same order.

The second term in Eq. (5) is the regularization term which ensures a certain smoothness to the material parameters along the abdominal wall. In this term, $\alpha_{j}$ is the regularization parameter. The regularization term also embeds prior information about the material parameter distribution into the inverse problem.

In this paper, we consider two different types of regularization. The Total Variation Diminishing (TVD) (Issa \& Javareshkian, 1998) is given by 
where $c$ is a small non-zero number that ensures that Eq. (6) is differentiable when $\nabla \beta_{j}=0$. TVD suppresses oscillations in the reconstructions and smooths the solution for noisy data without penalizing large gradients in the reconstruction. It is able to detect sharp spatial changes in material parameters, and is robust to noise in a small convergence region.

The second regularization type implemented in the algorithm is Tikhonov $\left(H^{1}\right)$ (Engl et al., 1989), defined as

$$
R\left(\beta_{j}\right)=\int_{\Omega_{0}}\left|\nabla \beta_{j}\right|^{2} d \Omega_{0}
$$

In contrast to TVD, $H^{1}$ tends to smooth out sharp spatial changes in material parameters. However it is quite accurate is determining the spatial location of large changes in material parameters (Ito et al., 2011; Liu et al., 2013).

\subsection{Material property distribution in the abdominal wall}

Starting from the $3 \mathrm{D}$ coordinates of the fiducial markers in the reference state $(\mathrm{IAP}=0$ $\mathrm{mmHg}$, see Fig. 1) the geometry of the whole external abdominal cavity was reconstructed. The procedure was the same for both specimen. To do that, we used the 3D modelling software Rhinoceros (2014) to interpolate a surface through the fiducial points. The geometry was closed considering the geometrical reference of control points in the surgical table.

Once the geometry of the abdominal wall was completely defined, a finite element mesh was generated using $A B A Q U S^{\circledR}$ (Simulia., 2011). The mesh was constructed with 3D tetrahedral elements (C3D4H), with a mean of 19796 nodes and 87191 elements per specimen. We considered a constant thickness of $3 \mathrm{~mm}$ normal to the surface based on previous abdominal wall studies (Hernández et al., 2011). The thickness of the abdominal wall was covered by three elements (see Fig. 5 (b)). No muscle layer was distinguished along the thickness.

Boundary conditions reproducing the pneumoperitoneum tests were applied by clamping sides and back of the model. Displacement of the side nodes (on border of the model closest to the head and on the border closest to the hind legs) was observed to be negligible during the experiments (see Fig 4; side dots are circled). Therefore, the vertical displacement of these nodes was assumed to be zero. The mesh of the abdominal wall extended in the lateral directions all the way to the back of the animal, which was in contact with the surgical table. Nodes in contact with the surgical table were also assumed to be fixed. Both boundary conditions applied to the model can be seen in Fig. 5 painted in orange: antero-posterior sides, Fig. 5 (a); back, Fig. 5 (b). A final pressure of $12 \mathrm{mmHg}$ was considered along the interior elements of the geometry. 
The algorithm began with a homogeneous material property distributions with initial values of $\mu=7 \cdot 10^{-3} \mathrm{MPa}$ and $\gamma=1.4[-]$ (seed). These values were obtained from previous MonteCarlo simulations performed on the same model. During the solution of the inverse problem these parameters were allowed to vary in a range of $[0.0001-0.05] M P a$ for $\mu$ and $[0.0001-5]$ for $\gamma$ (we note that gamma is dimensionless).

Since $\mu$ governs the small strain behavior and $\gamma$ controls the exponential stiffening (see Fig. 3 ), each parameter was recovered using data from different levels of pressure. To determine the shear modulus $\mu$, experimental displacement field corresponding to the sixth level of pressure $(P=6 \mathrm{mmHg})$ was used. This level was regarded to be within the linear elastic range of the material, but as large as possible within that range in order to maximize measurement accuracy relative to measurement error. Once this parameter distribution was established, the nonlinear parameter distribution was obtained with the displacement data at the highest level of pressure $(P=12 \mathrm{mmHg})$. The regularization parameters for both $\mu$ and $\gamma$ are listed in Table 1.

This numerical process was conducted on the two tests per specimen in order to assess the repeatability of the results.

\section{$3 \quad$ Results}

The abdomen shape changed during the inflation test from a flat cylinder to an ovoid shape, as can be seen in Fig. 6 (a). Results of the inverse analysis were visualised with ParaView (Ahrens et al., 2005). In Fig. 6 (b) the colour scale represents the displacement value of fiducial markers, which constitute the primary input to the inverse problem. Furthermore, in Fig. 6 (c) we plot the predicted displacement field obtained after solving the inverse problem with the TVD regularization.

The accuracy of the final solution was examined by determining the error between the measured and predicted displacement field at the highest applied pressure $(12 \mathrm{mmHg})$. Additionally, the correlation of regions with higher material parameter values with the anatomical composition of the animal model wall was evaluated.

\subsection{Material parameter reconstructions}

The spatial distribution of the material parameters with the TVD regularization for the two tests conducted on each specimen are shown in Fig. 7: (a) Specimen 1, (b) Specimen 2. In the figure, left images represent the distribution for $\mu$ (calculated from displacements at $P=$ $6 \mathrm{mmHg}$ ) and right ones represent the distribution for $\gamma$ (calculated from displacements at $P=12 \mathrm{mmHg}$. Red zones represent a stiffer behaviour while blue zones have a more compliant 
response. Only the front surface is plotted here since it is the area where we have experimental data. The material parameters of the surface in contact with the table are fixed at their initial values.

In each reconstruction, we observe a wide red strip in the middle of the abdomen for the shear modulus. The nonlinear parameter showed a more uniform distribution along the surface, with values slightly higher in case of Specimen 1. We also note some spots with large shear modulus without an elevation in the nonlinear parameter.

Similar results were found for Specimen 1 when using $H^{1}$ regularization (shown in Fig. 8). With this regularization a smoother distribution for the shear modulus is found in all tests. The red strip in the middle of the abdomen appears to be slightly thinner and more regular than for the TVD case. In contrast to this, in Specimen 1 the zone with the larger value of $\gamma$ appears to be bigger with this regularization type.

\subsection{Accuracy of the solution}

In order to quantify the accuracy of the reconstructed material property distributions, we directly compare the experimental surface obtained from the coordinates of black dots measured by the cameras and the predicted surface resulting from the parameter distributions determined by inverse analysis. Thus, an error contour map has been calculated for each test and regularization type, so that the error committed between the experimental and numerical data in the frontal abdominal surface was shown. These maps were calculated for several levels of pressure but results from last level $(P=12 \mathrm{mmHg})$ have been chosen as reference to draw a comparison between models.

As comparison, we first compute this difference for the displacement field obtained by solving the forward elasticity problem using the initial guess, that is assuming homogeneous distribution of material parameters along the wall. Maps are shown in Fig. 9 for both specimens. We note that there are significant regions where this difference exceeds $6 \mathrm{~mm}$ in magnitude.

In Fig. 10 we plot the error contour maps with the predicted displacements resulting from the inverse analysis. TVD results are displayed in figures on the left and $H^{1}$ in figures on the right. We observe that these errors are low for both regularization types and in particular, significantly smaller than those found with the homogeneous distribution.

We also observe that regions with larger errors are close to the edges where we have no data, and where boundary conditions are applied. 


\subsection{Stress stretch behavior}

In order to graphically observe the different mechanical response between abdominal zones with distinct material parameters, we chose two points on the upper abdominal surface (one on the center and one on the side, see Fig. 11 for their locations) and plot their stretch stress curves.

The situation of the points in regard to the material parameter distributions are shown in Fig. 11 (a) and (b). Using these parameters, biaxial tension simulations were performed in order to obtain the theoretical stress-stretch curves (see Fig. 11 (c)). To do so, for each point and specimen, the four results (2 tests $\mathrm{x} 2$ regularization) were considered, and median and standard deviation were calculated (values in Table 2). According to this figure, mechanical differences between groups of muscles are easily seen: rectus abdominis + linea alba (point A) and external oblique + internal oblique + transversus (point B).

\section{Discussion \& Conclusions}

In this study, we presented a methodology to characterize in vivo the passive mechanical behaviour of the abdominal wall using an animal model. Starting from experimental tests we have recovered the displacement field of the wall in a non invasive way and we have reconstructed the whole wall geometry to create a 3D FE model. Assuming an incompressible hyperelastic isotropic model with a strain energy function depending on two parameters, we have reproduced numerically the experimental tests to obtain the distribution of these material parameters that best reproduce the experimental data. These values were calculated by inverse analysis and two different types of regularization were used for the analysis.

Material parameter reconstructions for both regularizations are given in Fig. 7 and Fig. 8 respectively. For both types, distributions of tests of the same specimen were found to be very similar, indicating that the test is repeatable. When specimens are compared, we see that parameter values are comparable between the two animals, but the spatial distributions of the parameters differ in their details (mainly in $\gamma$ reconstruction). This observation highlights the necessity of testing each individual specimen, though some of the differences may be attributable to a coarse spacing of the fiducial markers. We should not expect to resolve spacial features smaller than the spacing of the fiducial markers. The TVD regularization is found to introduce artifacts into the reconstructions, in the form of small islands of stiffness surrounding the measurement points. They can be observed in the $\mu$ distributions and they are considered a bad aspect of this regularization, since they introduce features below the resolution of the data, and further, we recognize that they correspond to no anatomical features. On the contrary, $H^{1}$ regularization does not introduce such artifacts. Rather, it smooths the distribution around the locations of the fiducial markers. Overall values

of the parameters are similar to the ones obtained with the TVD regularization, which proves that the results are robust with respect to the choice of regularization method. 

determined. The accuracy of both of these depend on experimental and analysis parameters. For example, the spatial resolution at which we can reconstruct the material properties is roughly equal to the distance between fiducial markers. We cannot detect a change that occurs on a scale smaller than this length. Further, the reconstructed modulus values are influenced by our assumed thickness of $3 \mathrm{~mm}$ for the abdominal wall.

The red strip found in the $\mu$ reconstructions indicates that this zone had a more rigid behaviour than the sides for low pressures. This is logical considering that this is the place where the rectus abdominis lies, together with the fascia and the linea alba, both of which greatly increase the rigidity of the tissue (Grässel et al., 2005).

To fully understand the implications of the material parameter distributions we have to consider the anatomical distribution of the abdominal wall in a New Zealand rabbit. In Fig. 12, the main abdominal muscles situated in different layers are represented: Rectus abdominis, external oblique (EO), internal oblique (IO) and transversus abdominis (TA). The points analized in section 3.3 and shown in Fig. 11 correspond to different muscles or muscle composites: Point $\mathrm{A}$ is on the rectus abdominis while Point $\mathrm{B}$ is situated on the EO-IO-TA composite. The mechanical differences found between these points, characterized by the differences in the mechanical parameters $\mathrm{mu}$ and gamma at these points, seem to be associated with the mechanical differences between muscle layers and could be use as a characterization of those layers (or composite layers) when working in in vivo conditions.

The stress stretch curves shown in Fig. 11 (c) provide useful mechanical information about zones of the abdomen, in a manner which may be more informative than the numerical values of mu and gamma. For instance, the curves show the magnitude of stretch where the stiffening first occurs, and how far the more compliant material may be stretched before its tangent stiffness becomes equal to the stiffer material. This information may in future be applied in the operating room, when the mechanical response of the specific area (for example where a hernia has occurred) is needed. The low variation found between tests on same specimen gives a degree of confidence in the repeatability of the tests. Likewise, both specimens resulted in parameter distribution of the comparable magnitude and spatial distribution, which tends to buoy our confidence in the results. On the other hand, the slight differences found between specimens demonstrates the utility of using subject specific in vivo tests.

As an additional evaluation of the accuracy of the solution, predicted and measured surfaces were compared at the last level of pressure. When the forward solution from the initial guess of mechanical parameters was plotted (see Fig. 9), relatively large differences were found between the experimentally measured and the numerically predicted. This is logical since the forward problem assumes a homogeneous response of the abdominal wall, which means that same behaviour is supposed to be exhibited by all muscles. After the inverse analysis was performed, the material spatial variation of the material parameters is found to conform to the anatomical structure of the subject's abdomen. This spatial variation yields a numerically predicted deformed surface that agrees much more closely to the experimentally measured surface (Fig. 10) than did the orig- 

experimentally measured deformation surfaces occurs at the sides of the domain. These errors are consistent with a rotation or rolling of the subject about the cranio-caudal direction. Indeed, such a rotation was observed in the data at the higher pressure levels.

It is worth mentioning that this algorithm has been previously tested adding different levels of noise to the measured displacement field in order to assess the noise effect in the final inverse approach. In Gokhale et al. (2008) and Goenezen et al. (2011), authors guarantied the performance of the approach on synthetically generated displacement data up to $3 \%$ of noise. In this study the measurement technique here used can assure an accuracy of $1 \mathrm{~mm}$, which considering the $40 \mathrm{~mm}$ of maximum displacement, represents a noise of $2.5 \%$.

Some limitations of this study should be mentioned here. The 3D FE model has been reconstructed from point displacements measured at several different pressures during the pneumoperitoneum experiment. Increasing the number of pressure levels used may further improve the accuracy of the results. Likewise, widening the surface tracked during the tests would help to increase the amount of information captured during each deformation, and also extend that information closer to the edges of the computational domain for the FE model. We have considered a constant thickness along the model of the wall but it may be better obtain by ultrasound in a future. Regarding the choice of the material model, an isotropic incompressible hyperelastic material model was used here to reproduce the abdominal wall behavior. Presumably models that account for different material layers and/or anisotropy would be capable of more accurately predicting abdominal wall deformation. As these models depend on a much greater number of material parameters, however, a much larger number of deformation measurements would be required to determine all the parameters within those models. It's not clear that such models would improve with the limited amount of information available to this study. The fact that the present simpler model captured the observed behavior of the subject indicates that for these deformations, the present models are sufficient. Finally, since the ultimate goal of this work is the implementation of this methodology in human herniated cases, it would be very interesting to investigate how the hernia mechanically affects to the surrounding tissue, in order to be able to extrapolate the response of the healthy tissue from the external measurement. An in vivo study of a surgical mesh placed in an herniated abdomen could be also very interesting as a future step.

Despite these limitations, we have identified and presented a methodology to characterize in vivo the passive mechanical behaviour of the abdominal wall by using a completely non invasive measurement approach. This method obtains mechanical information from the pneumoperitoneum, a device step frequently used in surgery. The method is able to distinguish zones with different stiffness in the abdomen and quantify their mechanical parameters. The use of inverse analysis allows us to simultaneously quantify the in vivo tissue mechanical properties within each zone, and build a patient-specific computational model of the abdominal wall that may be used to simulate different loading states and potentially other deformations and manipulations. In closing, 
we observe that with the exception of the use of the pneumoperitoneum, which is otherwise commonly used in abdominal procedures, the methodology described here in entirely non invasive.

\section{Conflict of interest statement}

No financial support from other organizations unless the cited in the acknowledgements section, nor personal relationships, nor people, nor organisms biased the development of this research work.

\section{Acknowledgments}

The authors gratefully acknowledge research support from the Spanish Ministry of Economy and Competitiveness through the research project DPI2014-54981R and the ICTS NANBIOSIS, specifically by the Tissue \& Scaffold Characterization Unit (U13) of CIBER-BBN. We also thank the Spanish Ministry of Economy and Competitiveness for the financial support to R. Simón-Allué through the grant BES-2012-053422 and the mobility fellowships EEBB-I-14-08368 and EEBB-I-15-09811. Finally, support from the NSF (Grant Nos. 1148124, and 1148111) is also acknowledged.

\section{References}

Guide for the care and use of laboratory animals. Institute of Laboratory Animal Resources. Committee on Care and Use of Laboratory Animals and National Institutes of Health (US). Division of Research Resources, Washington (DC), 8th edn.

J. Ahrens, et al. (2005). ParaView: An End-User Tool for Large Data Visualization, Visualization Handbook. Elsevier.

J. M. Bellón (2007). 'Abdominal Wall Hernia Repair: A Comparison of Sepramesh and Parietex Composite Mesh in a Rabbit Hernia Model'. Journal of the American College of Surgeons 205:192-198.

H. Ben Abdelounis, et al. (2013). 'Effect of two loading rates on the elasticity of the human anterior rectus sheath'. Journal of the Mechanical Behavior of Biomedical Materials 20:1-5.

P. J. Blatz, et al. (1969). 'On the Mechanical Behavior of Elastic Animal Tissue'. Transactions of The Society of Rheology 13(1):83-102.

D. Chauvet, et al. (2015). 'In Vivo Measurement of Brain Tumor Elasticity Using Intraoperative Shear Wave Elastography'. Ultraschall in der Medizin .

W. Cobb, et al. (2005). 'The argument for lightweight polypropylene mesh in hernia repair'. Surg Innov 12:63-69. 

of porcine linea alba'. Journal of the Mechanical Behavior of Biomedical Materials 41:68-82. H. W. Engl, et al. (1989). 'Convergence rates for Tikhonov regularisation of non-linear ill-posed problems'. Inverse Problems 5(4):523.

S. Goenezen, et al. (2011). 'Solution of the nonlinear elasticity imaging inverse problem: The incompressible case'. Computer Methods in Applied Mechanics and Engineering 200:1406 1420.

S. Goenezen, et al. (2012). 'Linear and nonlinear elastic modulus imaging: an application to breast cancer diagnosis.'. IEEE transactions on medical imaging 31(8):1628-37.

N. H. Gokhale, et al. (2008). 'Solution of the nonlinear elasticity imaging inverse problem: the compressible case'. Inverse Problems 24(4):045010.

D. Grässel, et al. (2005). 'Anisotropy of human linea alba: A biomechanical study'. Journal of Surgical Research 124(1):118-125.

B. Hernández, et al. (2011). 'Mechanical and histological characterization of the abdominal muscle. A previous step to modelling hernia surgery'. Journal of the Mechanical Behavior of Biomedical Materials 4(3):392-404.

R. I. Issa \& M. H. Javareshkian (1998). 'Pressure-Based Compressible Calculation Method Utilizing Total Variation Diminishing Schemes'. The American Institute of Aeronautics and Astronautics Journal 36(9).

K. Ito, et al. (2011). 'A regularization parameter for nonsmooth Tikhonov regularization'. SIAM Journal on Scientific Computing 33:4015-38.

M. Kirilova, et al. (2011). 'Experimental study of the mechanical properties of human abdominal fascia'. Medical engineering \& physics 33(1):1-6.

U. Klinge, et al. (2002). 'Impact of polymer pore size on the interfaces scar formation in a rat model'. Journal of Surgical Research 103:208-214.

J. Kybic \& D. Smutek (2005). 'Estimating Elastic Properties of Tissues from Standard 2D Ultrasound Images'. In S. Y. Walker, W. F. ; Emelianov (ed.), Medical Imaging 2005: Ultrasonic Imaging and Signal Processing, vol. 5750, pp. 184-195, San Diego, CA.

I. Lichtenstein, et al. (1989). 'The tension-free hernioplasty'. The American Journal of Surgery 2(157):188-193.

J. Liu, et al. (2013). 'A novel combined regularization algorithm of total variation and Tikhonov regularization for open electrical impedance tomography'. Physiological Measurement 34(7):823-838.

M. Lyons, et al. (2014). 'Mechanical characterisation of porcine rectus sheath under uniaxial and biaxial tension'. Journal of biomechanics 47(8):1876-84.

P. Martins, et al. (2012). 'Mechanical characterization and constitutive modelling of the damage process in rectus sheath'. Journal of the Mechanical Behavior of Biomedical Materials 8:111122.

A. Nava, et al. (2008). 'In vivo mechanical characterization of human liver'. Medical Image Analysis 12(2):203-216.

T. Nilsson (1982). 'Biomechanical studies of rabbit abdominal wall. Part I. The mechanical properties of specimens from different anatomical positions'. Journal of Biomechanics 15(2):123-129.

J. Ophir, et al. (1991). 'Elastography - A Quantitative Method for Imaging the Elasticity of Biological Tissues'. Ultrasonic Imaging 13:111-134. 
H. Paajanen \& H. Hemunen (2004). 'Long-term pain and recurrence after repair of ventral incisional hernias by open mesh: clinical and MRI study'. Langenbeck's Archives of Surgery 389:366-370.

PhotoModeler (2013). PhotoModeler Scanner. Eos Systems Inc, Vancouver, Canada.

F. Podwojewski, et al. (2014). 'Mechanical response of human abdominal walls ex vivo: Effect of an incisional hernia and a mesh repair'. Journal of the Mechanical Behavior of Biomedical Materials 38:126-33.

Rhinoceros (2014). Rhinoceros 3D. McNeel Europe, Barcelona, Spain.

H. Rivaz, et al. (2013). 'Ultrasound elastography using multiple images'. Medical image analysis 18(2):314-329.

R. Simón-Allué, et al. (2015). 'Developing a new methodology to characterize in vivo the passive mechanical behavior of abdominal wall on an animal model'. Journal of the Mechanical Behavior of Biomedical Materials 51:40-49.

Simulia. (2011). Abaqus user's guide, v. 6.11. Dassault Systemes Simulia Corp., Providence, RI, USA.

C. Song, et al. (2006). 'Mechanical properties of the human abdominal wall measured in vivo during insufflation for laparoscopic surgery'. Surgical Endoscopy 20(6):987-990.

C. Szymczak, et al. (2012). 'Investigation of abdomen surface deformation due to life excitation: implications for implant selection and orientation in laparoscopic ventral hernia repair'. Clinical biomechanics (Bristol, Avon) 27(2):105-10.

D. Tran, et al. (2014). 'Contribution of the skin, rectus abdominis and their sheaths to the structural response of the abdominal wall ex vivo'. Journal of biomechanics 47(12):3056-63.

D. Tran, et al. (2016). 'Abdominal wall muscle elasticity and abdomen local stiffness on healthy volunteers during various physiological activities'. Journal of the Mechanical Behavior of Biomedical Materials 60:451-459.

C. Zhu, et al. (1997). 'L-BFGS-B: Algorithm 778: L-BFGS-B, FORTRAN routines for large scale bound constrained optimization'. ACM Transactions on Mathematical Software 23(4):550560. 


\begin{tabular}{lccc}
\hline Regularization type & $\alpha_{\mu}$ & $\alpha_{\gamma}$ & $\mathrm{c}$ \\
\hline$H^{1}$ (Eq. 7) & 0.2 & 1 & - \\
TVD (Eq. 6) & 0.01 & 0.1 & 0.125 \\
\hline
\end{tabular}

Table 1

Regularization parameters for $\mu$ and $\gamma$, for material property reconstructions. 


\begin{tabular}{|l|cc|cc|cc|cc|}
\cline { 2 - 10 } \multicolumn{1}{c|}{} & \multicolumn{4}{c|}{ Specimen 1 } & \multicolumn{4}{c|}{ Specimen 2 } \\
\hline \multirow{2}{*}{ Point } & \multicolumn{2}{|c|}{ A } & \multicolumn{2}{|c|}{ B } & \multicolumn{2}{c|}{ A } & \multicolumn{2}{c|}{ B } \\
\cline { 2 - 10 } & Mean & Std & Mean & Std & Mean & Std & Mean & Std \\
\hline$\mu[\mathrm{MPa}]$ & $5.0 \cdot 10^{-2}$ & 0 & $3.90 \cdot 10^{-3}$ & $1.08 \cdot 10^{-3}$ & $4.99 \cdot 10^{-2}$ & $6.74 \cdot 10^{-5}$ & $9.65 \cdot 10^{-3}$ & $7.72 \cdot 10^{-4}$ \\
\hline$\gamma[-]$ & 2.25 & 0.35 & 1.83 & 0.19 & 1.55 & 0.11 & 1.57 & 0.14 \\
\hline
\end{tabular}

Table 2

Material parameters of points A and B for both specimen (see Fig. 11). 


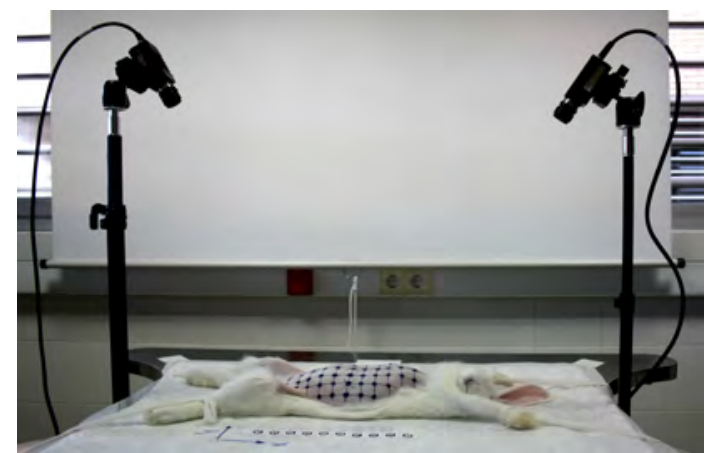

(a)

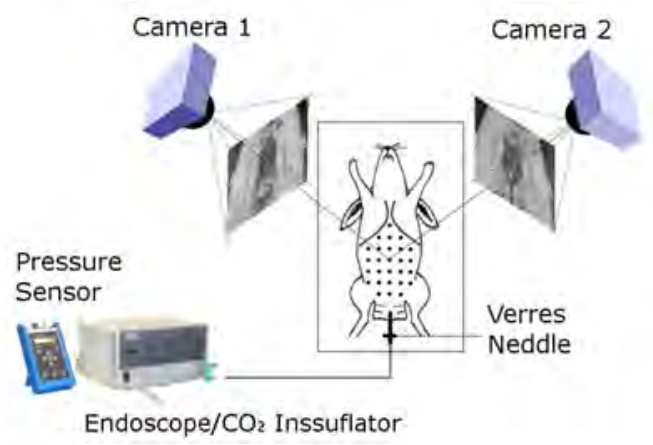

(b)

Fig. 1. Lab experimental setup: (a) Real disposition of the cameras with regard to the specimen. (b) Schematic diagram of the whole setup. 


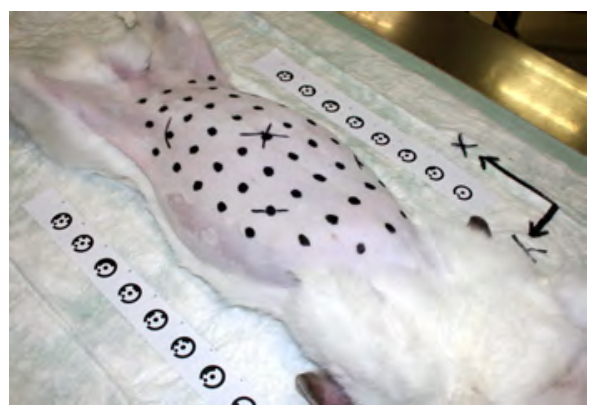

(a) $P=0 \mathrm{mmHg}$

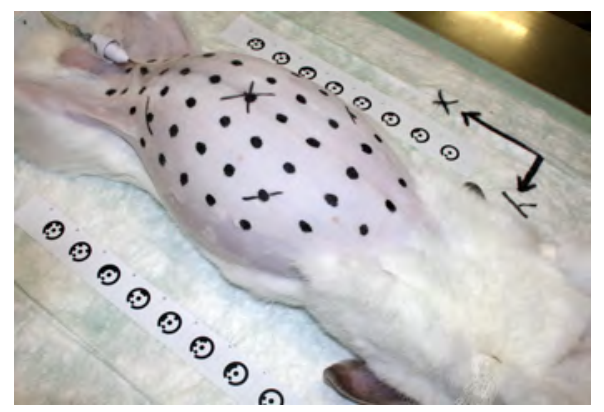

(b) $P=12 m m H g$

Fig. 2. Abdominal surface of the specimen in the initial (a) and final (b) instant of the inflation test. 


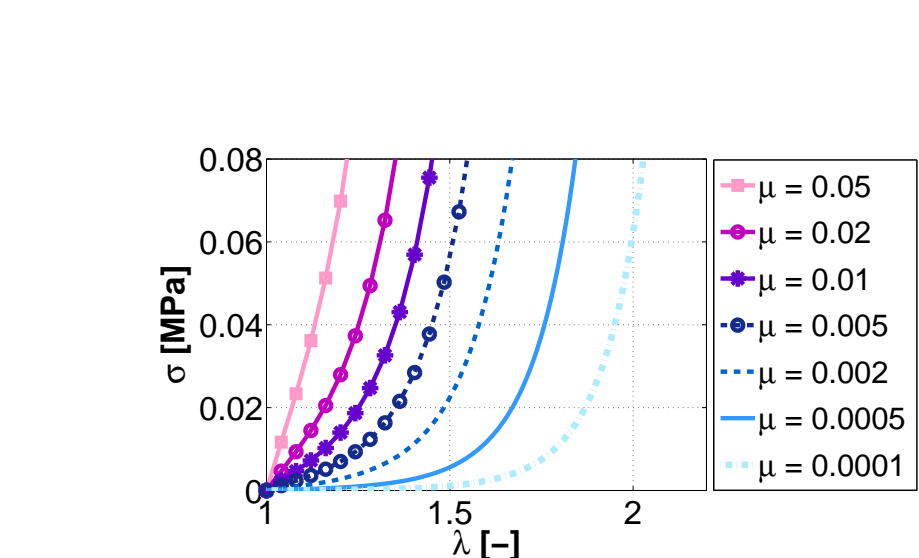

15

16

17

18

19

20

21

22

23

24

25

26

27

28

29

30

31

32

33

34

35

36

37

38

39

40

41

42

43

44

45

46

47

48

49

50

51

52

53

54

55

56

57

58

59

60

61

62

63

64

65

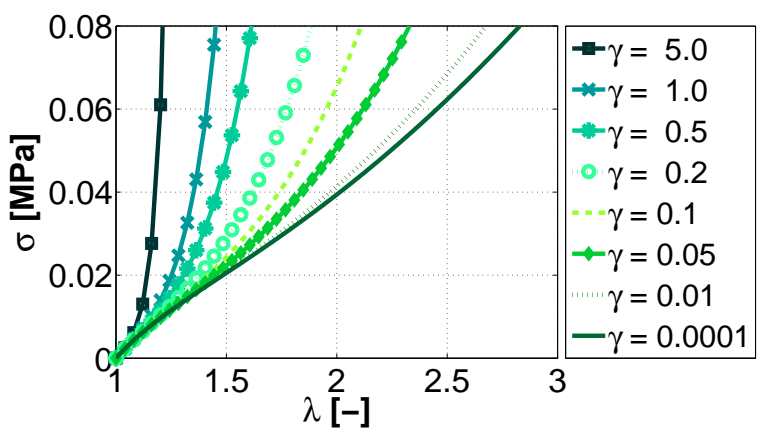

(a) Cauchy stress versus stretch for $\gamma=1[-]$ (b) Cauchy stress versus stretch for $\mu=$ $0.01 M P a$

Fig. 3. Range of the material parameters in stress stretch curves. 

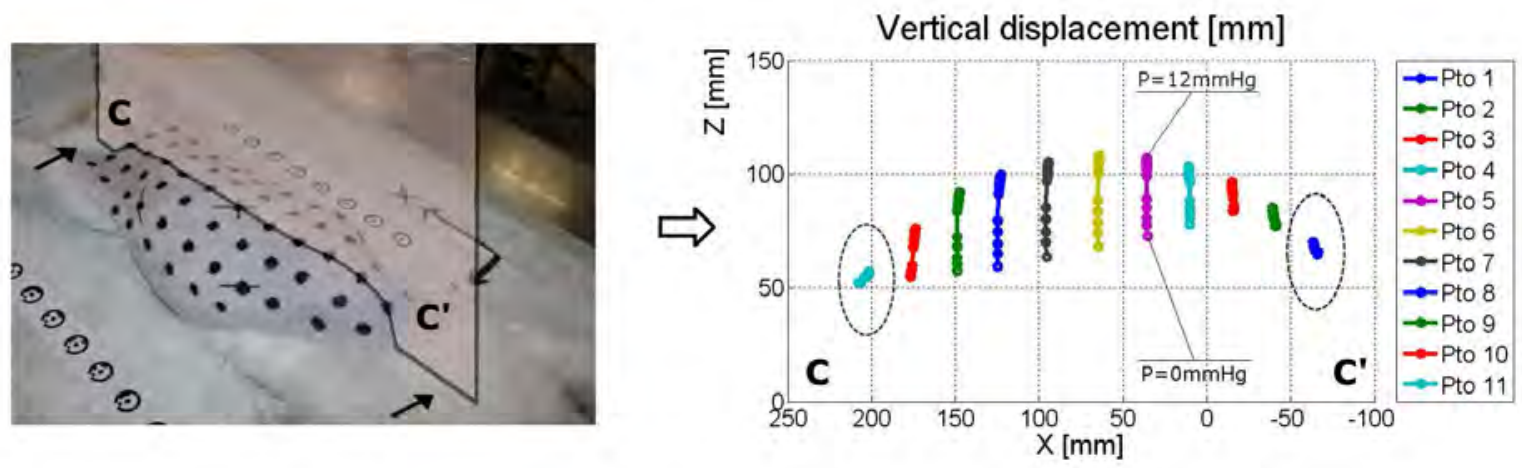

Fig. 4. Vertical tracking of the points that conform the line C-C/. 


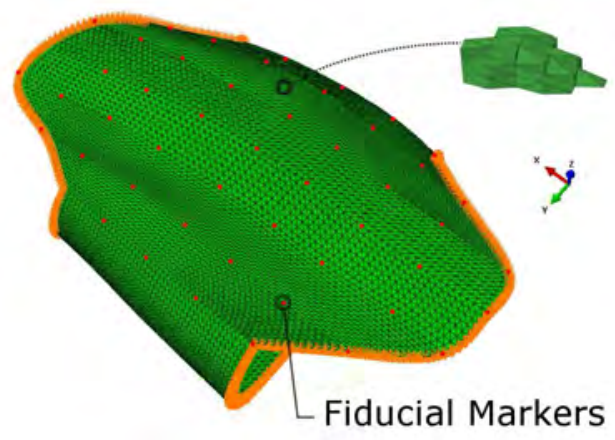

(a)

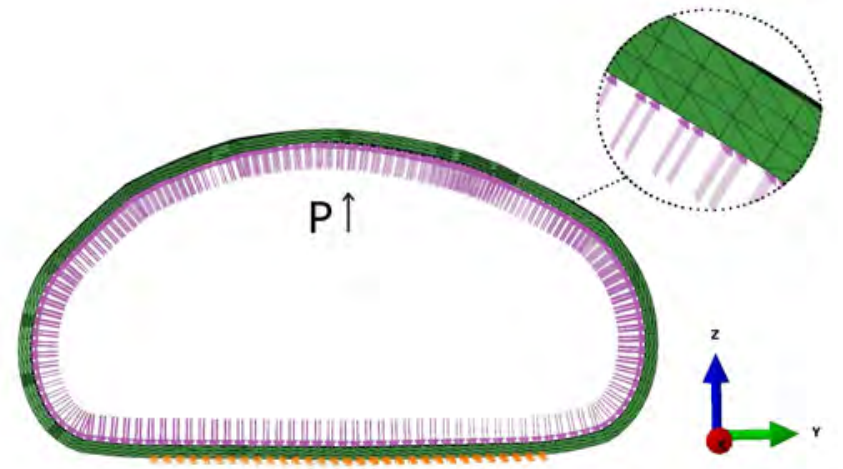

(b)

Fig. 5. Features of the abdominal wall mesh. (a) The fiducial markers are denoted by red points, and the nodes that were fixed are denoted by orange points. (b) Section view: the fixed nodes are indicated by orange dots, and the pressure field through purple arrows.

23

24

25

26

27

28

29

30

31

32

33

34

35

36

37

38

39

40

41

42

43

44

45

46

47

48

49

50

51

52

53

54

55

56

57

58

59

60

61

62

63

64

65 


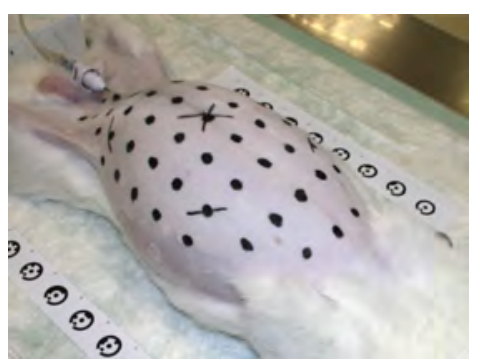

(a)

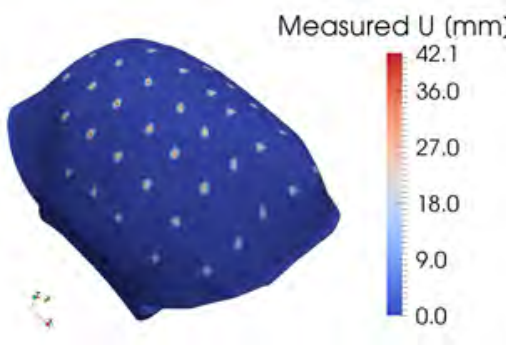

(b)

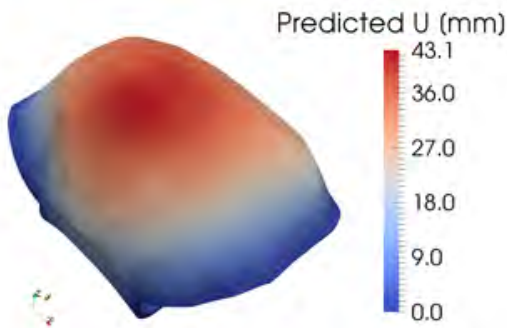

(c)

Fig. 6. (a) Deformed shape of the abdominal wall at $P=12 \mathrm{mmHg}$. (b) Measured vertical displacement of the fiducial markers, plotted on the predicted deformed shape. (c) Predicted vertical displacement field obtained after solving the inverse problem with the TVD regularization, plotted on the predicted deformed shape. 

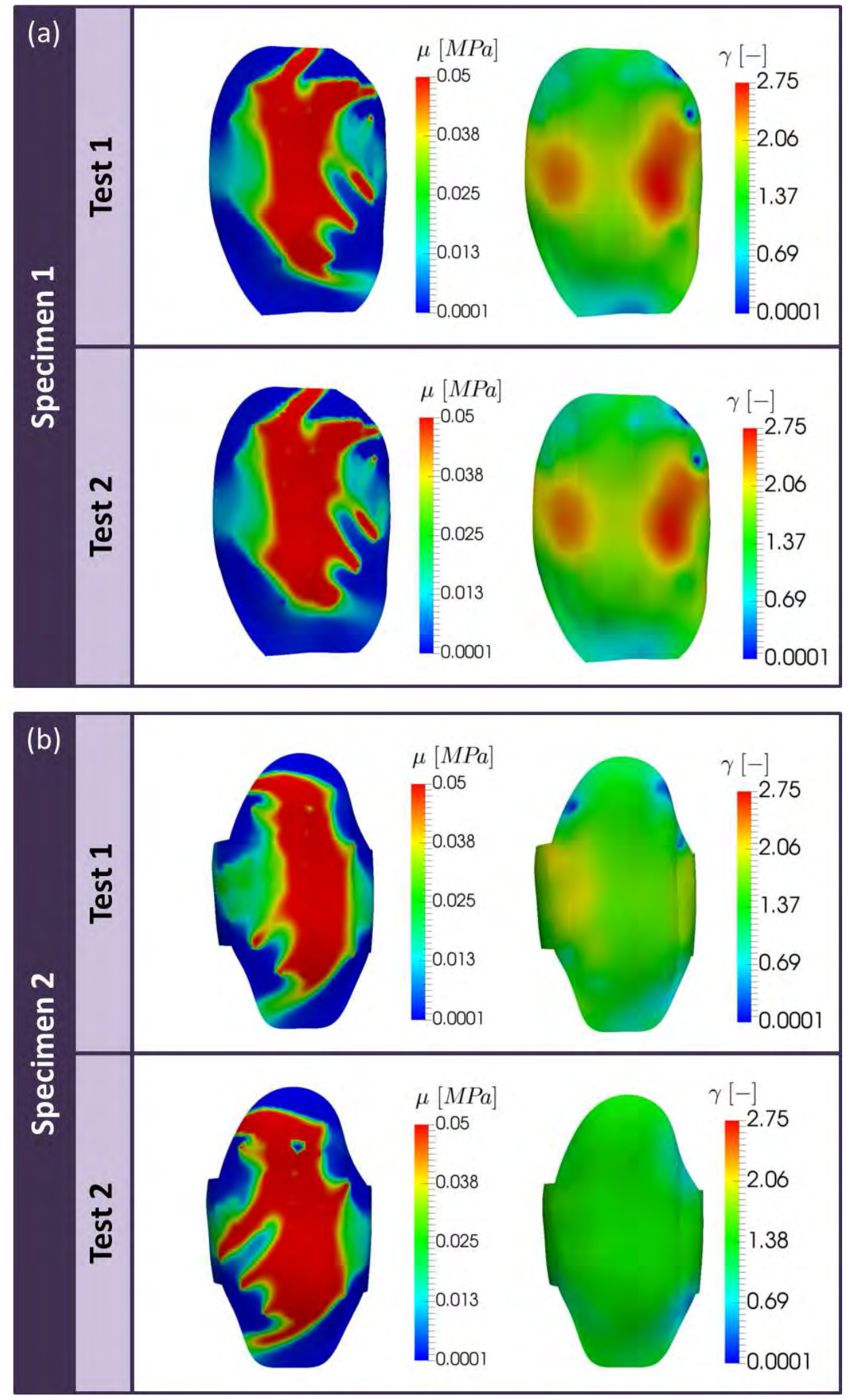

Fig. 7. Reconstruction of the material parameters after solving the inverse problem with TVD regularization: (a) Results from the specimen 1; (b) results from specimen 2. 

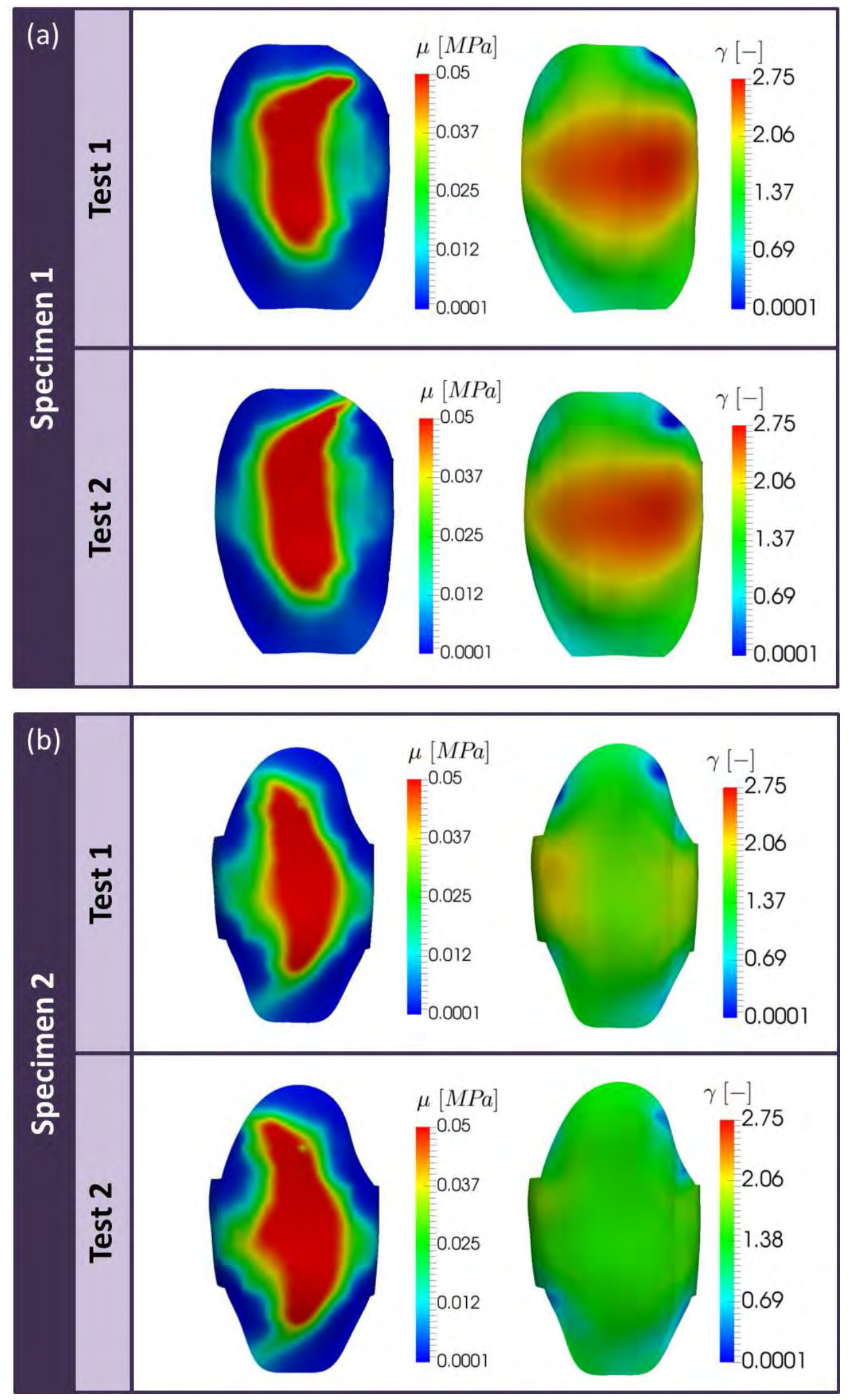

57

58

59

60

61

62

63 


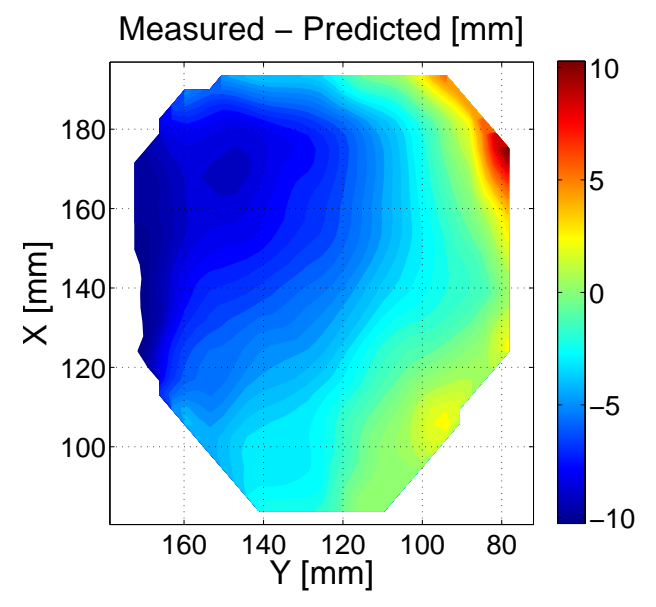

(a) Specimen 1

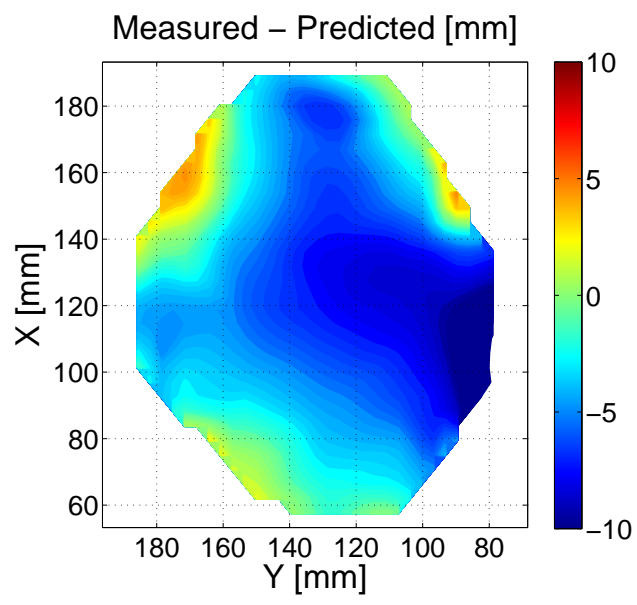

(b) Specimen 2

Fig. 9. Error contour maps for the forward problem (Homogeneous response). Difference in mm between the measured and predicted surface at $12 \mathrm{mmHg}$. 

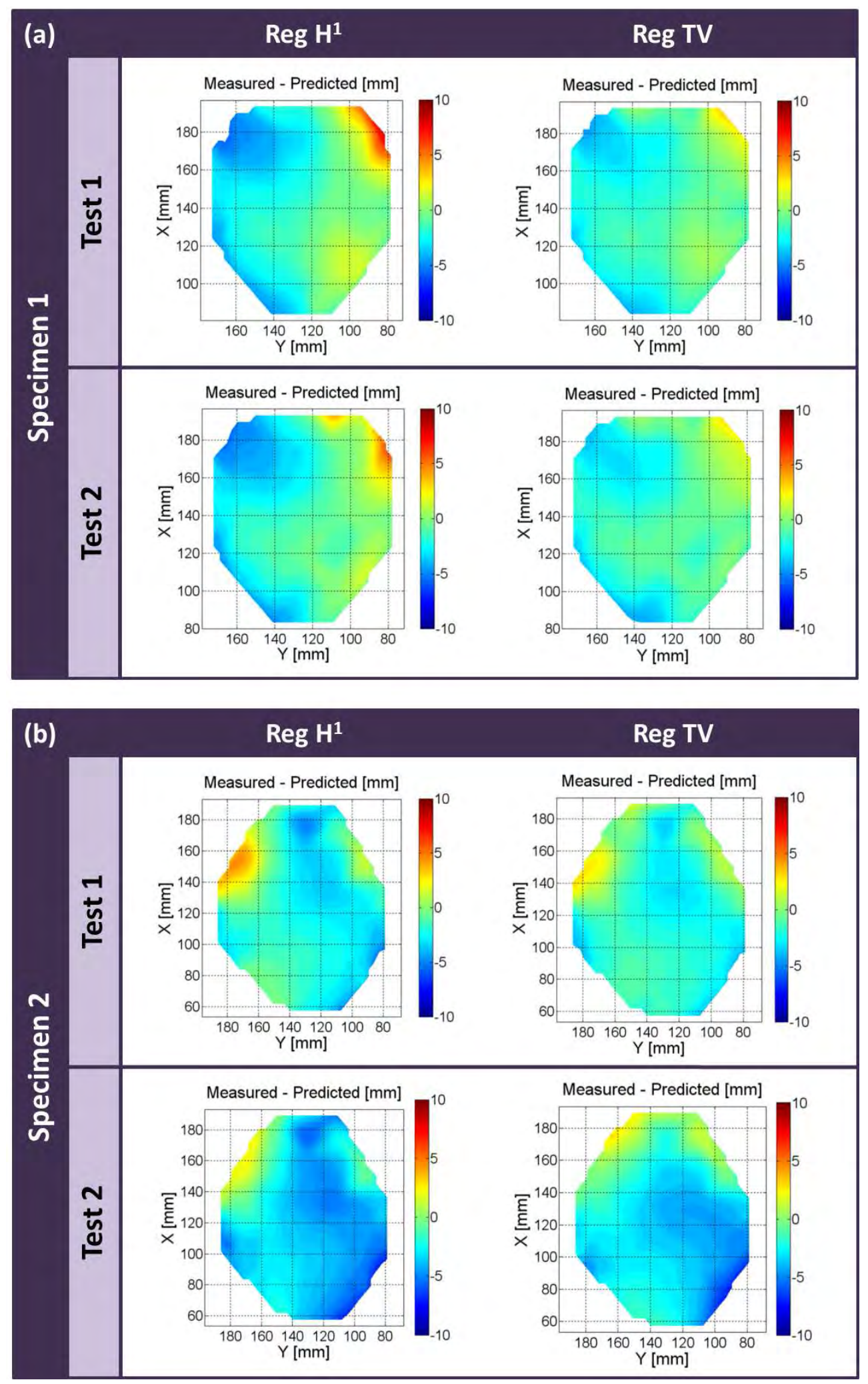

Fig. 10. Error contour maps after solving inverse problem. Difference in mm between the measured and predicted surface at $12 \mathrm{mmHg}$. 


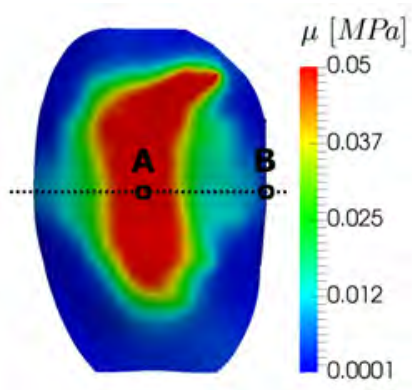

(a) Shear Modulus $\mu$.

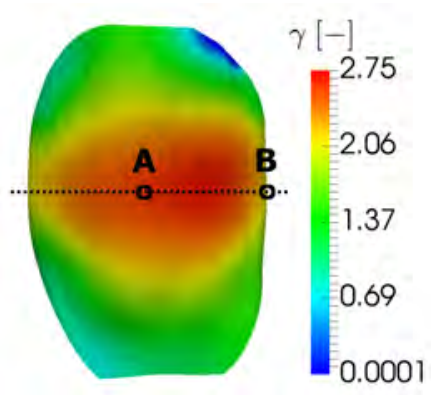

(b) Nonlinear parameter $\gamma$.

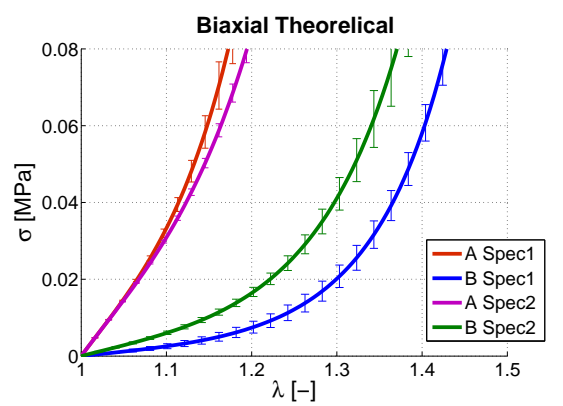

(c) Stress versus stretch

Fig. 11. Mechanical differences between two areas of the reconstructed material properties along the transversal direction. 


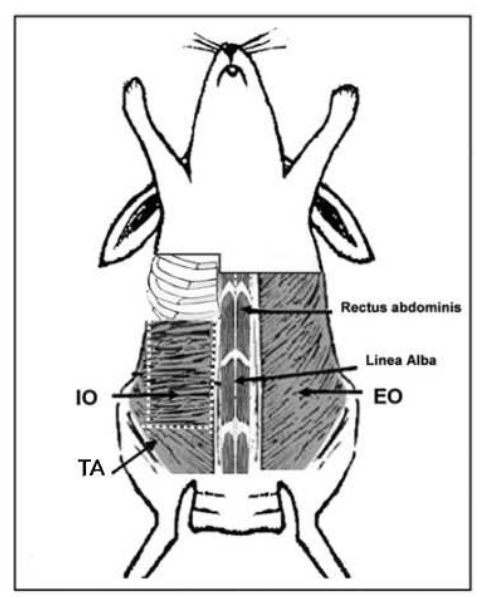

Fig. 12. Model of the muscle composition in the abdominal wall of the New Zealand white rabbit (EO: external oblique; IO: internal oblique; TA: transversus abdominis). Image obtained from literature (Hernández et al., 2011). 